\title{
Topography and morphometry of the subarcuate canal
}

\author{
J. Skrzat ${ }^{1}$, B. Leszczyński², M. Kozerska ${ }^{1}$, A. Wróbel² \\ ${ }^{1}$ Department of Anatomy, Jagiellonian University, Collegium Medicum, Krakow, Poland \\ ${ }^{2}$ Department of Medical Physics, Institute of Physics, Jagiellonian University, Krakow, Poland
}

[Received 3 April 2013; Accepted 7 May 2013]

The current study shows in a close-up view anatomical relationship between the subarcuate canal and the osseous labyrinth. For this purpose we used micro-computed tomography which allowed performing three-dimensional reconstruction of the subarcuate canal and gave adequate data for estimation its diameter across its course. The diameter of the middle part (the most uniform) of the subarcuate canal varied from $0.28 \mathrm{~mm}$ to $0.46 \mathrm{~mm}$. Hence, we calculated the centre of mass for each cross-section of the separated subarcuate canal. This procedure helped us to visualise trajectory of the subarcuate canal and its spatial orientation within the petrous bone. From our data we concluded that subarcuate canals revealed not well defined trajectories and their spatial orientation varied across the studied temporal bones. (Folia Morphol 2013; 72, 4: 357-361)

Key words: petromastoid canal, petrous bone, micro-computed tomography

\section{INTRODUCTION}

The subarcuate canal (petromastoid canal) originates from the subarcuate fossa and runs in a posterior, lateral and superior direction. It courses between 2 limbs of the superior semicircular canal of the labyrinth. The subarcuate canal connects the mastoid cells with the posterior cranial fossa. Through its lumen runs the subarcuate artery (a branch from the anterior inferior cerebellar artery or from the internal auditory artery), vein and prolongation of the dura mater $[7,9]$.

The subarcuate canal was first described by Mouret and Rouviere in 1904 [5, 7]. Since that time this bony canal was rarely the subject of morphological analysis. Small size was a serious obstacle in visualising its structure and locality in radiographs. Application of high resolution computed tomography (CT) allowed imaging more accurately osseous components housed within the petrous bone [5, 7]. This gave a solid background to perform comparative study of the subarcuate canal and to determine its morphological variability [1]. To the best of our knowledge, volume rendered three-dimensional (3D) $\mathrm{CT}$ images of the subarcuate canal have not been published before. Thus, we used micro-CT to investigate morphological features of the subarcuate canal in humans and present its topography in 3D space. Applied modality is superior to conventional CT imaging because it allows observing anatomical structures with extremely high resolution with pixel sizes of the cross-sections in the micrometre range.

The aim of this study was to represent pictorially relationship between the subarcuate canal and the superior semicircular canal. Contrary to other studies, we evaluated how varies diameter of the subarcuate canal and represented numerically its trajectory.

\section{MATERIALS AND METHODS}

Morphological study of anatomy and topography of the subarcuate canal was performed on 5 dry temporal bones, labelled as Tb1, Tb2, Tb3, Tb4, and Tb5. All the bones were well preserved and presented normal anatomy. Three samples (Tb1, Tb4, Tb5) derived 
from adult individuals of female sex, and 2 samples (Tb2, Tb3) derived from infant skulls of unknown sex. Their sex could not have been established only on the morphological features of the temporal bones and the skulls did not remain in intact condition. However, the lack of this information is without meaning for the process of imaging and 3D reconstruction of the anatomical structures being the subject of the current study.

The petrous part of the temporal bone was dissected and scanned with the micro-CT scanner (Skyscan 1172, N.V., Aartselaar, Belgium). The scanner was equipped with the X-ray detector: 11 Megapixel (4024 × 2680 in total; $4000 \times 2400$ effective), 12-bit digital X-ray camera with $24 \times 36 \mathrm{~mm}$ field of view. The $X$-ray source voltage was set to $80 \mathrm{kV}$ and current to $100 \mu \mathrm{A}$. The projection images were acquired over an angular range of $180^{\circ}$ with an angular step of $0.5^{\circ}$. In the resultant images pixel size was $27 \mu \mathrm{m}$. Projections were captured along the long axis of the petrous bone and reconstructed using a software NRECON ver. 1.6.5 SkyScan (CTVox, CTAn, CTVol), based on the Feldkamp algorithm.

Volume rendering was used as a technique to display a 2D projection of a 3D discretely sampled data set produced by the micro-CT scanner and visualised in CTVox application. Basing on the histogram display of the intensities present in the volume data we adjusted interactively the transfer function that maps voxel intensities to provide the best perception of the observed structures. In that way we visualised different material components of the object having differing $X$-ray opacity. Visualisation of the air spaces was performed in the CTVox software because it allows for exploitation of the blending mode. By modifying the opacity we could control the visibility of the voxels. Next, the region of interest which contained only the subarcuate canal was defined in each micro-CT scan and binarised with the aid of the threshold algorithm implemented in CTAn application. From the obtained dataset, the 3D model of the subarcuate canal was created and visualised with the aid of the CTVol application. Using CTAn application we calculated structure thickness distribution of the reconstructed canal in 3D space. In our study this parameter expressed the diameter of the subarcuate canal. For the obtained data, we fitted Gaussian function which determined the mean diameter.

Hence, using free public domain image processing software "ImageJ" (http://imagej.nih.gov/ij) we calculated the centre of mass for each cross-section of the separated subarcuate canal. This procedure helped us to visualise trajectory of the subarcuate canal and its spatial orientation within the petrous bone.

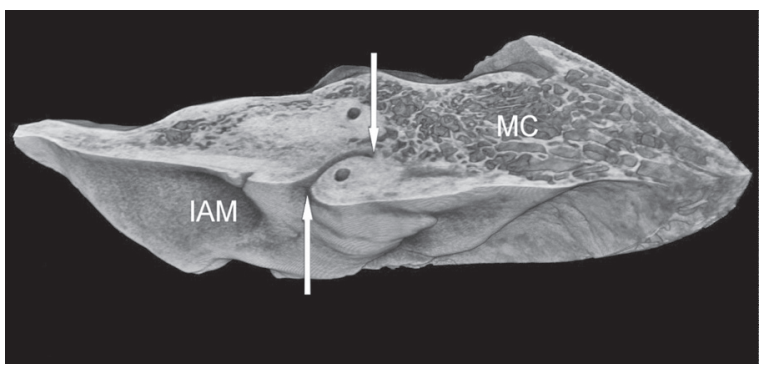

Figure 1. Horizontal section through the petrous bone reveals the inlet - subarcuate fossa (lower arrow) and outlet - mastoid antrum (upper arrow) of the subarcuate canal; IAM — internal acoustic meatus; MC — mastoid cells.

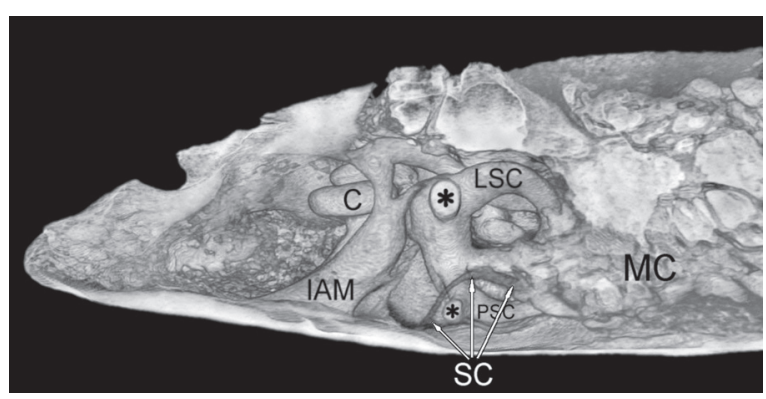

Figure 2. Volumetric reconstruction of the subarcuate canal (SC) showing its relationship to the superior semicircular canal (marked by asterisks) and other components of the petrous bone; IAM internal acoustic meatus; $\mathrm{C}$ - cochlea; PSC — posterior semicircular canal; LSC — lateral semicircular canal; MC — mastoid cells.

The study was conducted with approval (KBET/109/B/2012) of the Bioethics Committee of the Jagiellonian University.

\section{RESULTS}

Micro-CT scans of the petrous part of the temporal bone demonstrated presence of the subarcuate canals in all examined specimens which communicated the middle ear with the intracranial cavity. Volume reconstructions and planar sections of each sample revealed topography of the subarcuate canal and its relationship to the neighbouring osseous structures. Both the beginning and the end of the canal was easily identified in all samples. Overall morphology and the position of the subarcuate canal within the petrous bone is shown in Figure 1. However, small size of this canal forced to perform close-up reconstruction of the interior of the petrous bone. This was achieved by generation of volume rendered images with adjusted transfer function and viewed at different angles. The effect of this procedure in combination with virtual sectioning is presented in the Figures 2-4.

Values of the standard deviations indicate that precision of measurement was similar in all cases 


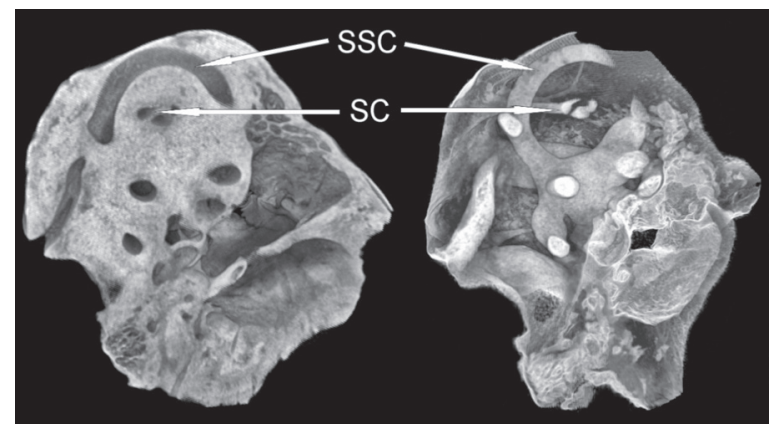

Figure 3. Volumetric reconstruction of the petrous bone dissected in the region of the superior semicircular canal (SSC) showing position and cross-section of the subarcuate canal (SC). Structures are rendered in low opacity (left image), and in high opacity (right image).

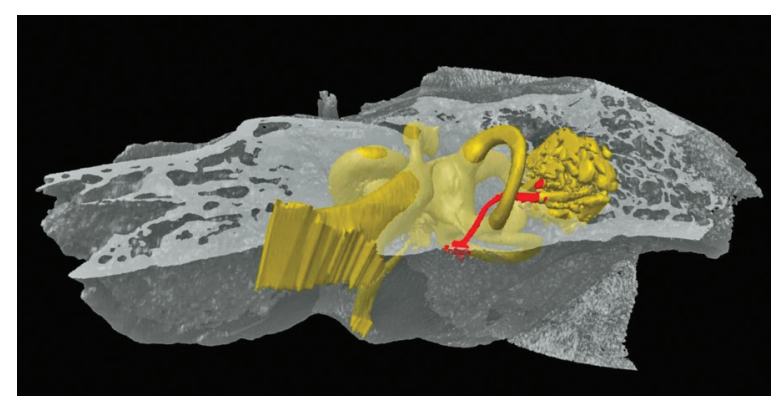

Figure 4. A model of the surface topography of the petrous bone in the horizontal section showing passage of the subarcuate canal (red) under the superior semicircular canal.

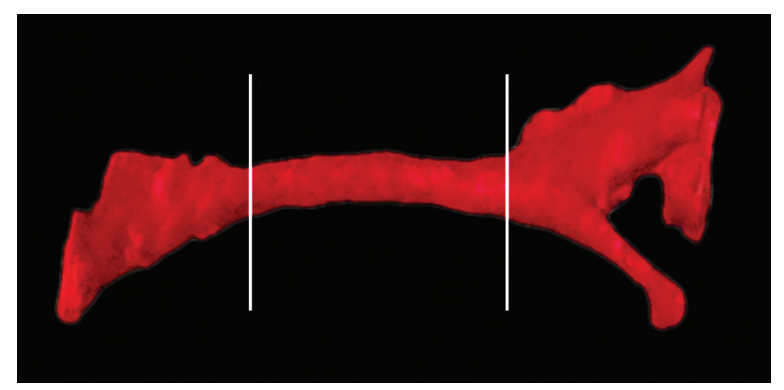

Figure 5. Computer model of the isolated subarcuate canal. Lines of orientation demarcate approximate portion of the canal for which mean diameter was calculated. and characterise an error of the mean diameter. The middle of the Gaussian distribution refers to the mean diameter of the subarcuate canal. Diameters below and above mean value correspond to the beginning and the ending of the canal. This is dictated by the morphology of the canal on the medial surface of the petrous bone where the canal opens (the inlet to the canal is a widened cleft), and its connection to mastoid cell where it also becomes wider or even bifurcated. The diameter of the middle part of the subarcuate canal varied from $0.28 \mathrm{~mm}$ to $0.46 \mathrm{~mm}$, and for all samples the average diameter was $0.44 \mathrm{~mm}$.

From the analysis of the mass centres distribution we concluded that the subarcuate canals revealed not well defined trajectories and their spatial orientation varied across the studied temporal bones. In all cases they presented anterior convex course but with variable curvature. Moreover the subarcuate canals inclined from the horizontal plane and were locally obliquely positioned in the 3D space (Figs. 5, 6). The most distinct patterns of the canals were presented in Figure 7.

\section{DISCUSSION}

Up till now, the anatomy of the subarcuate canal has been studied by conventional CT. We used micro-CT imaging to investigate anatomy and topography of the subarcuate canal and the neighbouring osseous components of the petrous bone. In the case of studying small osseous structures this modality provides accurate images and facilitates to construct detailed virtual models. Volume render images obtained from micro-CT scans provided excellent visual information on topography of the subarcuate canal and its spatial relationship to the surface of the petrous bone and inner components, e.g. the labyrinth.

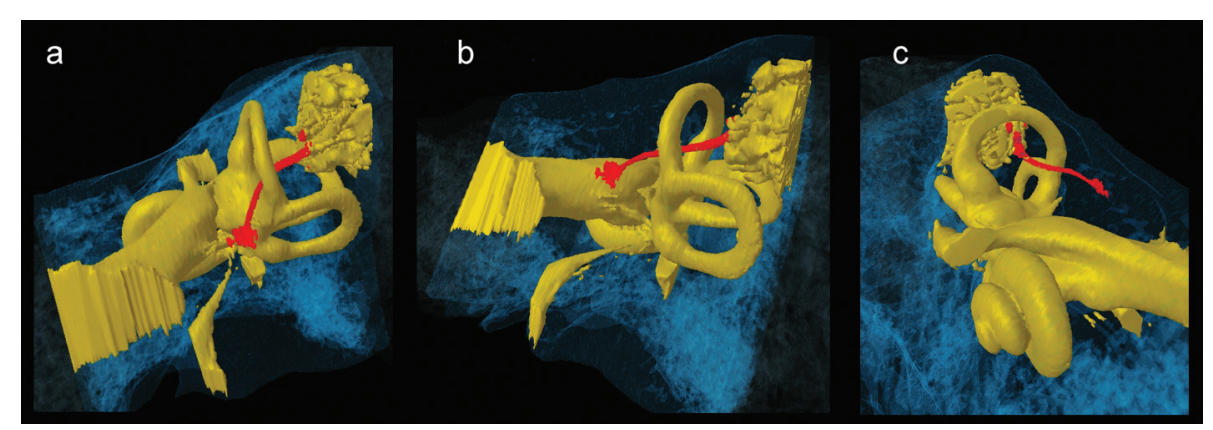

Figure 6. The 3D model of the osseous labyrinth (yellow) and the subarcuate canal (red) presented in different orientation; a — antero-medial view; b - medial view; c — latero-superior view. 


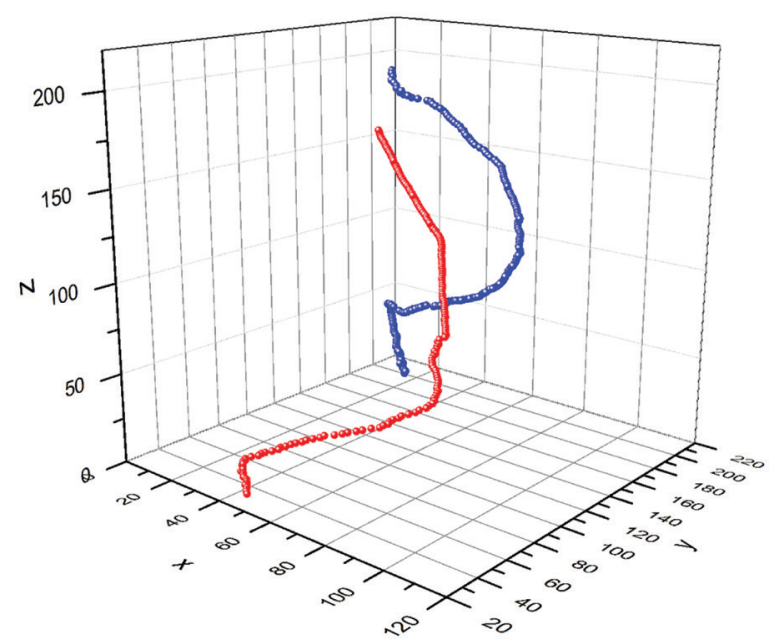

Figure 7. Trajectory of the subarcuate canals obtained from distribution of mass centres. Selected for two distinct patterns of the canal - convex (blue) and flat (red). The axis $(x, y, z)$ are presented in pixel units; 1 pixel $=27 \mu \mathrm{m}$.
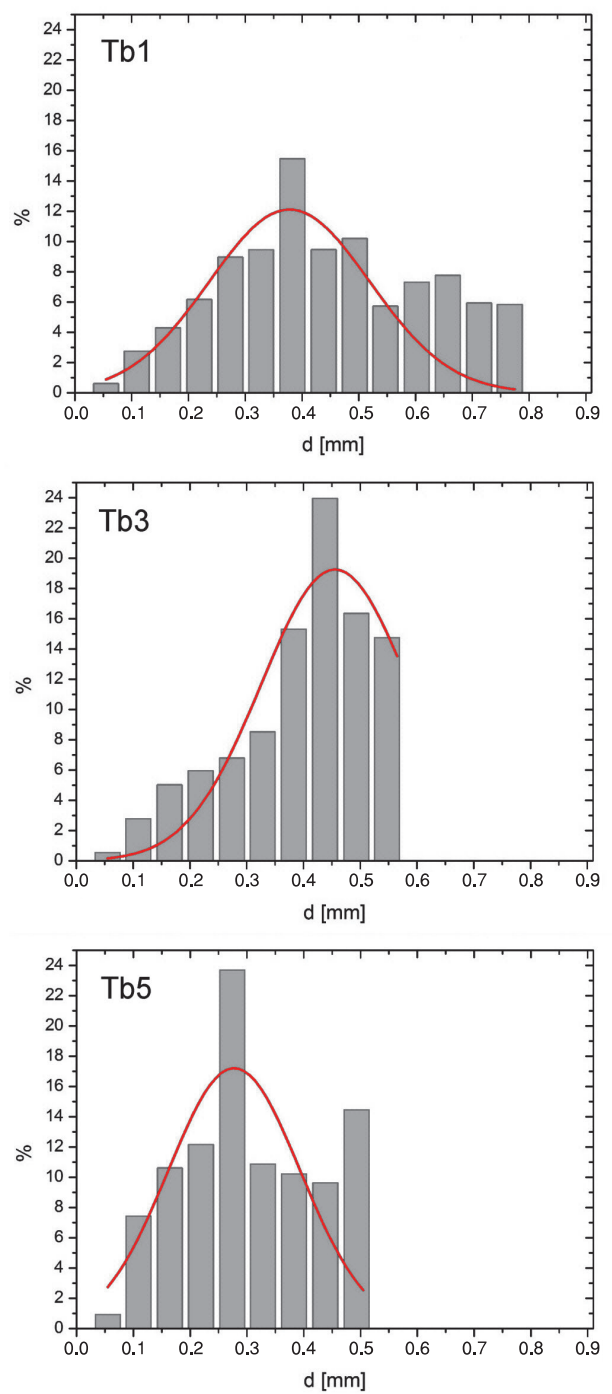

Hence, data from the micro-CT enabled to perform more sophisticated numerical analysis than clinical tomographic examination. We could measure variation of the diameter of the subarcuate canal along its course and assess its trajectory basing on distribution of centres of mass (Figs. 7, 8). In previous studies the width of the subarcuate canal was usually measured locally and characterised by a single value being the arithmetic mean. In the case of the subarcuate canal such a way of estimation seems to be biased because of its uneven shape. Both the beginning and the ending of the subarcuate canal are usually enlarged. This is not a uniform cylindrical object, except the middle portion where the canal is tubular in shape. Thereby, we presented the whole spectrum of the diameters which were encountered in each of the examined samples, and from these data the most
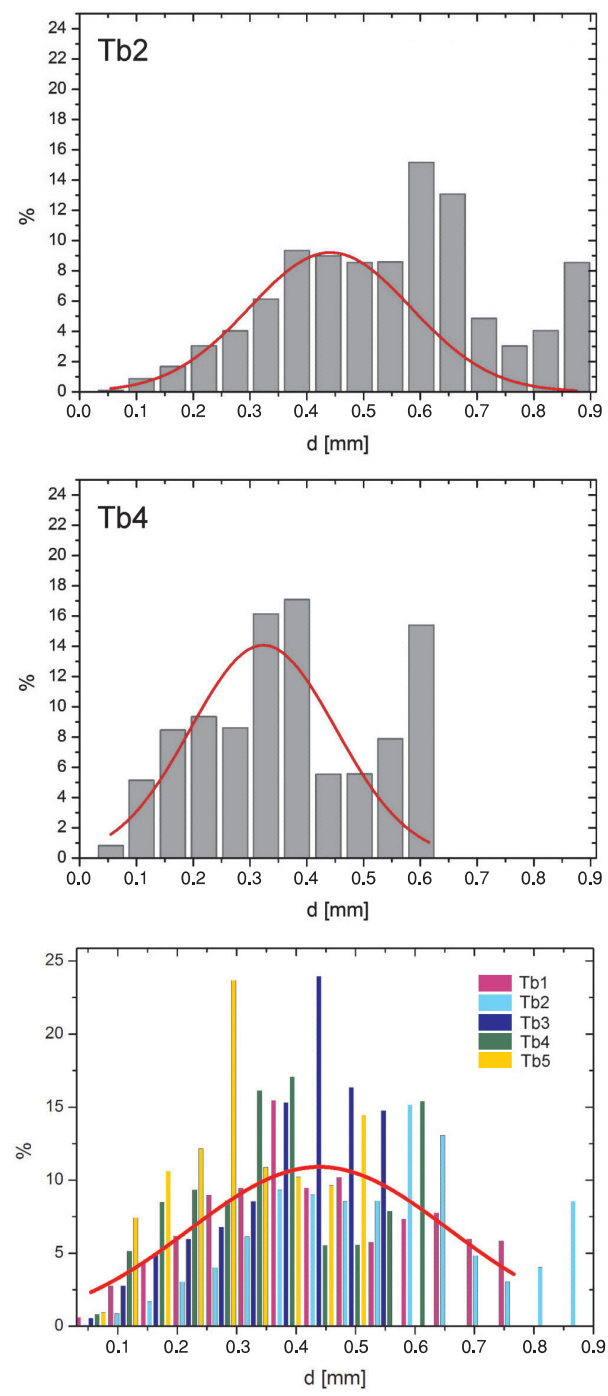

Figure 8. Variation of the diameter distributions estimated for the subarcuate canal in 5 temporal bones (Tb1-Tb5). The red lines represents Gaussian fit with mean value and standard deviation presented in Table 1. 
Table 1. Mean diameter of the subarcuate canal (xc) and standard deviation (SD).

\begin{tabular}{lc}
\hline Sample & xc [mm] \pm SD \\
\hline Tb1 & $0.38 \pm 0.14$ \\
Tb2 & $0.44 \pm 0.14$ \\
Tb3 & $0.46 \pm 0.13$ \\
Tb4 & $0.32 \pm 0.13$ \\
Tb5 & $0.28 \pm 0.12$ \\
All samples & $0.44 \pm 0.22$ \\
\hline
\end{tabular}

probable mean diameter of the subarcuate canal was established.

It is known that the subarcuate canal attains narrow and elongated appearance by 5 years of age. However, in children width of the subarcuate canal can be above $2 \mathrm{~mm}$, and it is still regarded as a normal anatomical variant [4-6]. According to Krombach et al. [3], mean diameter for the petromastoid canals measured for 10 men and 10 women of mean age 42 years was $0.51 \pm 0.04 \mathrm{~mm}$.

In other studies, the width of the subarcuate canal was found to be approximately $1 \mathrm{~mm}$ or it was larger (1.6-1.8 $\mathrm{mm}$ ) in singular cases. Enlargement of the canal was explained as the reason of the pneumatisation of the temporal bone [9]. In turn, Migirov and Kronenberg $[5,6]$ observed on CT images subarcuate canals which thickness varied from less than $0.5 \mathrm{~mm}$ to $1 \mathrm{~mm}$ or even greater. Steinbach et al. [8] estimated the middle diameter of the petromastoid canal which was $1.16 \pm 0.4 \mathrm{~mm}$, and varied from 0.25 to $2.5 \mathrm{~mm}$. They executed measurements on CT scans of normal temporal bones of 156 women and 160 men whose age varied from 19.6-84.2 years.

Our study performed on infant and adult temporal bones revealed that subarcuate canals showed mean diameters that ranged from 0.28 to $0.46 \mathrm{~mm}$. These values corresponds apparently with the width of the canals estimated from CT. Although we examined very small number of the temporal bones (only 5 samples) we noticed that our results fit to general tendency suggesting that thicker subarcuate canals are typical for children, whereas thinner occur more frequently in adults. In our study the subarcuate canals showing mean diameter equal $0.44 \mathrm{~mm}$ and $0.46 \mathrm{~mm}$ were of children, whereas the canals which mean diameter was $0.28,0.32$ and $0.38 \mathrm{~mm}$ belonged to adult individuals.

From the utilitarian point of view measurements of the subarcuate canal performed on the classic CT scans can be adequate for clinical procedures. Taking into account non-uniform morphology and trajectory of this canal further comparative and quantitative studies should be continued using modern explorative techniques. For time being, we proposed morphometrical analysis of the subarcuate canal in 3D space using structure thickness distribution to evaluate its mean diameter, and calculating centres of mass to reconstruct canal's trajectory.

Current literature delivers only a small piece of information on variation in the course of the subarcuate canal, its shape and spatial orientation $[2,5,6]$. Detailed comparative study on morphological variability and topography of the subarcuate canal could be beneficial for otolaryngologists because this canal is regarded as the potential route in transmission infections from middle ear into the intracranial cavity.

\section{ACKNOWLEDGEMENTS}

The research was carried out with the equipment purchased thanks to the financial support of the European Regional Development Fund in the framework of the Polish Innovation Economy Operational Program (contract no. POIG.02.01.00-12-023/08).

\section{REFERENCES}

1. Akyol Y, Galheigo D, Massimore M, Fatterpekar G (2011) Subarcuate artery and canal: an important anatomic variant. J Comput Assist Tomogr, 35: 688-689.

2. Grammatica A, Alicandri-Ciufelli M, Molteni G, Marchioni D, Presutti L (2010) Subarcuate canal and artery: a case report. Surg Radiol Anat, 32: 171-174.

3. Krombach GA, Schmitz-Rode T, Prescher A, DiMartino E, Weidner J, Günther RW (2002) The petromastoid canal on computed tomography. Eur Radiol, 12: 2770-2775.

4. Leung JY, Ishak GE (2010) Prominent subarcuate canal in children: a normal variant. Pediatr Radiol, 40 (suppl. 1): 161.

5. Migirov L, Kronenberg J (2006) Radiology of the petromastoid canal. Otol Neurotol, 27: 410-413.

6. Migirov L, Kronenberg J (2009) Petromastoid canal and cochlear aqueduct in cochlear implant candidates. Otolaryngol Head Neck Surgery, 140: 419-422.

7. Proctor B (1983) The petromastoid canal. Ann Otol Rhinol Laryngol, 92: 640-644.

8. Steinbach S, Fitzthum A, Reiser M, Hundt W (2009) Der petromastoidale Kanal. Eine computertomographische Untersuchung. HNO, 57: 142-145.

9. Tekdemir I, Aslan A, Elhan A (1999) The subarcuate canaliculus and its artery: a radioanatomical study. Ann Anat, 181: 207-211. 